\title{
The IAU 2009 system of astronomical constants: the report of the IAU working group on numerical standards for Fundamental Astronomy
}

\author{
Brian Luzum • Nicole Capitaine • Agnès Fienga • William Folkner • \\ Toshio Fukushima • James Hilton • Catherine Hohenkerk • George Krasinsky • \\ Gérard Petit · Elena Pitjeva · Michael Soffel · Patrick Wallace
}

Received: 20 December 2010 / Accepted: 30 April 2011 / Published online: 10 July 2011

(C) The Author(s) 2011. This article is published with open access at Springerlink.com

\begin{abstract}
In the 2006-2009 triennium, the International Astronomical Union (IAU) Working Group on Numerical Standards for Fundamental Astronomy determined a list of Current Best Estimates (CBEs). The IAU 2009 Resolution B2 adopted these CBEs as the IAU
\end{abstract}

George Krasinsky was recently deceased.

B. Luzum ( $\varangle) \cdot$ J. Hilton

U.S. Naval Observatory, Washington, DC, USA

e-mail: brian.luzum@usno.navy.mil

N. Capitaine

SYRTE, Observatoire de Paris, CNRS, UPMC, Paris, France

A. Fienga

Institut UTINAM, Université de Franche-Comté,

CNRS-UMR 6123, Besançon, France

W. Folkner

Jet Propulsion Laboratory, Pasadena, CA, USA

T. Fukushima

National Astronomical Observatory, Tokyo, Japan

C. Hohenkerk

HM Nautical Almanac Office, Taunton, UK

G. Krasinsky · E. Pitjeva

Institute of Applied Astronomy, RAS, St. Petersburg, Russia

G. Petit

Bureau International des Poids et Mesures, Sèvres, France

M. Soffel

Dresden Technical University, Dresden, Germany

P. Wallace

Rutherford Appleton Laboratory, Chilton, UK 
(2009) System of Astronomical Constants. Additional work continues to define the process of updating the CBEs and creating a standard electronic document.

Keywords Numerical standards · Fundamental Astronomy · Fundamental constants

\section{Introduction}

The International Astronomical Union (IAU) Working Group (WG) on Numerical Standards for Fundamental Astronomy is tasked with updating the IAU Current Best Estimates (CBEs), conforming with the IAU Resolutions, the International Earth Rotation and Reference Systems Service (IERS) Conventions and Système International d'Unités (SI) whenever possible. To achieve this, the WG closely cooperates with IAU Commissions 4 and 52, the IERS, and the Bureau International des Poids et Mesures (BIPM) Consultative Committee for Units.

This is the third IAU WG to be tasked with producing CBEs and is adding to the legacy of the two previous WGs. The first Sub-group on Numerical Standards of the IAU WG on Astronomical Standards was headed by E.M. Standish and the WG report (Standish 1995) established the rules that are still used today. For instance, this group (1) decided on the two-tiered approach to the astronomical constants that we are currently using (i.e. having both an official system of constants and a set of CBEs), (2) specified the content of the file of the current best estimates, and (3) created the first CBEs for a list of IAU constants.

This work was continued by T. Fukushima and his IAU WG on Astronomical Standards (Fukushima 2000, 2002). Many of the updates concerned work on constants in a general relativistic framework and improved estimates of the precession constant. This revised list of CBEs remained the currently adopted list of IAU CBEs until the IAU 2009 General Assembly. The excellent work of both these WGs has established the precedent and allows us to improve incrementally the values for which there are now better estimates.

There were several issues addressed by the WG over the triennium. The key items include

- selecting the best numerical values for the CBEs and the required criteria to be fulfilled to become a CBE;

- selecting the most appropriate classification and grouping of these constants;

- selecting the information to be provided in the Table of CBEs;

- selecting the mechanism for keeping the CBEs current; and

- whether to update the IAU System of Astronomical Constants.

There have been extensive discussions of the pertinent topics by e-mail. In addition, the WG was able to hold meetings at the 2007 and 2008 Journées "Systèmes de référence spatiotemporels" held in Meudon, France and Dresden, Germany, respectively.

\section{IAU 2009 Resolution B2}

The CBEs assembled by this WG provide a marked improvement over the IAU (1976) System of Astronomical Constants (IAU 1976) for a number of reasons. The most obvious reason is that in the intervening years, the estimates of many of the values have improved significantly. Also, over the years, additional values are now considered to be an integral part of the system 
of constants, mostly due to resolutions adopted by scientific bodies such as the IAU General Assembly. As a result, the Working Group proposed that the CBEs be adopted as the IAU (2009) System of Astronomical Constants. This was accomplished when Resolution B2 was passed by the 2009 IAU General Assembly. The text of this resolution is provided in the Appendix.

Table 1 is a reproduction of the values on 2009 August 13 that were adopted as the IAU (2009) System of Astronomical Constants. Unless otherwise noted, the constants should be considered to be in terms of the Système International d'Unités (SI). The terminology "XXXcompatible", depending on the coordinate time being used, follows the recommendations of the IAU Commission 52 Relativity in Fundamental Astronomy (RIFA) (see Klioner et al. 2010). Note that the terminology "unscaled" can also be used for both "TCB-compatible" and "TCG-compatible" values.

The names of the categories for the constants have been changed to better reflect the structure of the new system. The concept of "Defining Constant" was kept but was split into "Natural" (consistent with natural laws) and "Auxiliary" (consistent with conventionally adopted theory). "Natural Measurable Constants" was used for constants of nature that can be measured. The term "Body Constants" was used to describe the category containing the constants associated with solar system bodies. The category "Initial Values at J2000.0" contains a constant with a value chosen as a starting value for a specific theory adopted by the international community. The category "Other Constants" contains values that do not belong in any of the other categories and are kept for historical reasons.

\subsection{Notes on Table 1}

a. The Gaussian gravitational constant, $k$, is listed as an auxiliary defining constant as it continues to be used to define the relationship between $a u$ and $G M_{\mathrm{S}}$. This value from the IAU 1976 System of Astronomical Constants is retained in Table 1 and has been used to derive the $G M_{\mathrm{S}}$ value given in Table 1 from the DE421 value of the $a u$ (Folkner et al. 2008). However, other estimates of $G M_{\mathrm{S}}$ can be determined directly by fitting modern planetary ephemerides (e.g. INPOP08, DE423, EPM2008) to observations. See discussion below regarding the Gaussian gravitation constant, $k$, and heliocentric gravitation constant, $G M_{\mathrm{S}}$.

b. This constant comes from the expression $\mathrm{TDB}=\mathrm{TCB}-L_{\mathrm{B}} \times\left(\mathrm{JD}_{\mathrm{TCB}}-\mathrm{T}_{0}\right) \times 86400+$ $\mathrm{TDB}_{0}$, where $\mathrm{T}_{0}=2443144.5003725$.

c. This constant comes from the IAU 2000 Resolution B1.8 expression $\theta(U T 1)=$ $2 \pi(0.7790572732640+1.00273781191135448 \times($ Julian UT1 date -2451545.0$))$.

d. The value for $a u$ is TDB-compatible. An accepted definition for the TCB-compatible value of $a u$ is still under discussion.

e. All values of the masses from Mars to Eris are the sum of the masses of the celestial body and its satellite(s).

f. The values for $a_{\mathrm{E}}$ and $J_{2}$ are "zero tide" values (see IERS Conventions for an explanation of the terminology). Values according to other conventions can be found in Groten (2000).

g. $\omega$ is a nominal value and was chosen to have the number of significant digits limited to those for which the value can be considered constant.

h. $\epsilon_{J 2000}$ is a component of the IAU 2006 precession model, which includes expressions that are time dependent. 


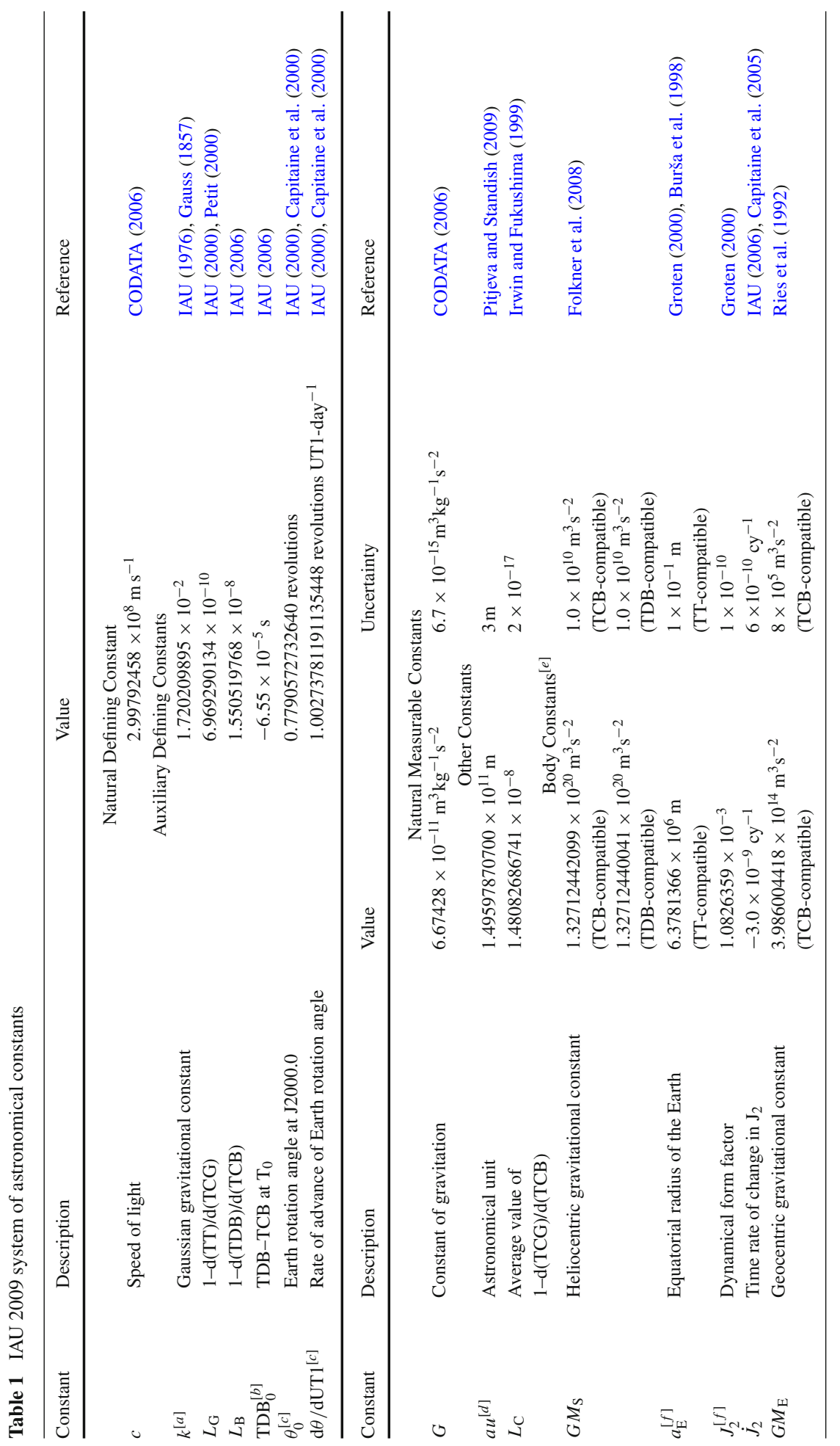




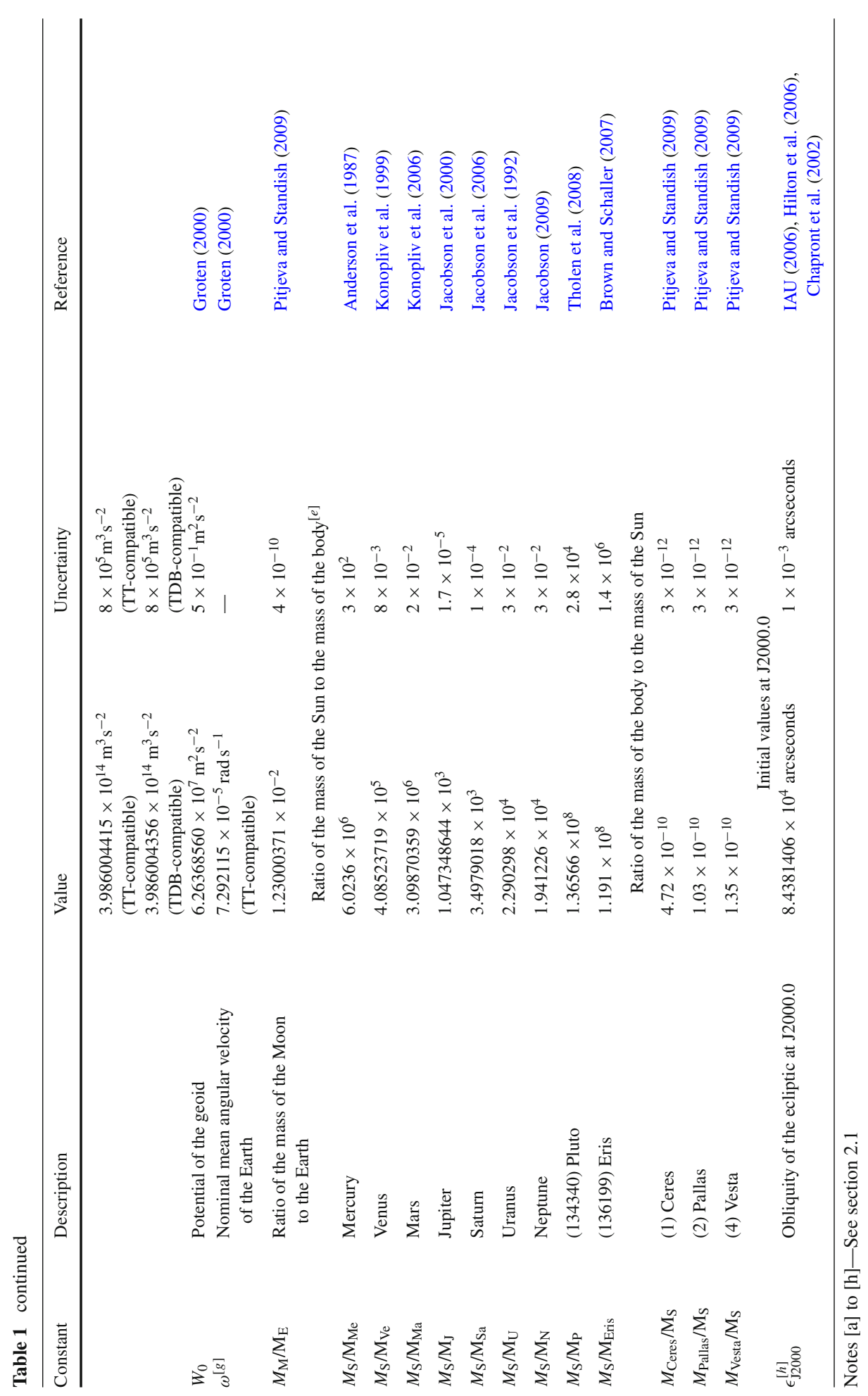




\section{Discussion}

This section provides background information regarding the selection of the defining constant or current best estimate. Any ancillary information or unresolved concerns regarding these numbers are also delineated here. One constant no longer included in this list is the rate of precession. This constant has been removed from the list since it is no longer appropriate as it is only one term of the IAU 2006 precession model.

Speed of light, $c$

The speed of light is a defining value for the IAU and the IERS. The reference to the value has been updated from CODATA 1998 (Mohr and Taylor 2000) to CODATA 2006 (Mohr et al. 2008) to reflect the use of the value of this constant by the current set of CODATA constants.

\section{Gaussian gravitation constant, $k$}

The Gaussian gravitation constant remains a defining constant of the IAU and was used for the definition of the value of $a u$ that is given in Table 1 (Pitjeva and Standish 2009). For users who need a consistent value for the heliocentric gravitational constant, $k$ should also be used to derive $G M_{\mathrm{S}}$ through the equation $a u^{3} k^{2} / D^{2}=G M_{\mathrm{S}}$ where $D$ is one day of $86400 \mathrm{~s}$. Note that estimates can now be determined directly by fitting modern planetary ephemerides to observations.

There is a difference in opinion regarding the use of $k$. Some would use $k$ in the traditional way along with a measured astronomical unit, $a u$, to calculate the heliocentric gravitation constant, $G M_{\mathrm{S}}$. Others believe that $a u$ should become a defining constant, which would fundamentally change the status of $k$. Although $k$ has been retained to be consistent with historical systems, it is expected that a recommendation will be discussed at the 2012 IAU General Assembly that will resolve this issue.

$L_{\mathrm{G}}$

The value for $L_{\mathrm{G}}$ is such that the mean rate of TT is close to the mean rate of the proper time of an observer located on the rotating geoid. It is specified in IAU 2000 Resolution B1.9 as a defining constant. The value for $L_{\mathrm{G}}$ is taken from Petit (2000).

$L_{\mathrm{B}}$

The value for $L_{\mathrm{B}}$ is specified in IAU 2006 Resolution B3 as a defining constant. The fixed value for $L_{\mathrm{B}}$ was derived in 2005 from the expression $L_{\mathrm{B}}=L_{\mathrm{C}}+L_{\mathrm{G}}-L_{\mathrm{C}} \times L_{\mathrm{G}}$, where $L_{\mathrm{G}}$ is given in IAU Resolution B1.9 (2000) and $L_{\mathrm{C}}$ has been determined (Irwin and Fukushima 1999) using the JPL ephemeris DE405. When using the DE405, the defining $L_{\mathrm{B}}$ value effectively eliminates a linear drift between TDB and TT, evaluated at the geocenter. When realizing TCB using other ephemerides, the difference between TDB and TT, evaluated at the geocenter, may include some linear drift, not expected to exceed 1 ns per year.

$T D B-T C B$ at $T_{0}, T D B_{0}$

The difference between TDB and TCB at $\mathrm{T}_{0}(=2443144.5003725), \mathrm{TDB}_{0}$, is specified in IAU 2006 Resolution B3 as a defining constant. This constant comes from the expression $\mathrm{TDB}=\mathrm{TCB}-\mathrm{L}_{\mathrm{B}} \times\left(\mathrm{JD}_{\mathrm{TCB}}-\mathrm{T}_{0}\right) \times 86400+\mathrm{TDB}_{0}$. The value for $\mathrm{TDB}_{0}$ is chosen to provide consistency with the TDB-TT formula of Fairhead and Bretagnon (1990). 
ERA at J2000.0, $\theta_{0}$, and rate of advance of ERA, $d \theta / d U T 1$

The values for the Earth rotation angle at J2000.0, $\theta_{0}$, and the rate of advance of the Earth rotation angle $\mathrm{d} \theta / \mathrm{dUT} 1$, are specified in IAU 2000 Resolution B1.8 as defining constants. These constants come from the expression $\theta(U T 1)=2 \pi(0.7790572732640+$ $1.00273781191135448 \times($ Julian UT1 date -2451545.0$))$. The values for these constants are taken from Capitaine et al. (2000).

\section{Constant of gravitation, $G$}

The value for the constant of gravitation, $G$, has been changed from the CODATA 1998 (Mohr and Taylor 2000) value to the value adopted by CODATA 2006 (Mohr et al. 2008).

\section{Astronomical unit, au}

The current best estimate for the astronomical unit, $a u$, has been taken from Pitjeva and Standish (2009) and is an average of recent estimates for the $a u$ defined by $k$. Note that this value is TDB-compatible and that an accepted definition for the TCB-compatible value of $a u$ is still under discussion.

$L_{\mathrm{C}}$

The value for $L_{C}$ is taken from Irwin and Fukushima (1999) and is the most recent published determination. It is based on the DE405. Note that before 2006, $L_{C}$ was used to compute $L_{\mathrm{B}}$ but since the adoption of IAU 2006 Resolution B3, that is no longer true. Before IAU 2006, $L_{\mathrm{B}}$ was computed as $L_{\mathrm{B}}=L_{\mathrm{C}}+L_{\mathrm{G}}-L_{\mathrm{C}} \times L_{\mathrm{G}}$. This relation does not hold [exactly] between the defining values of $L_{\mathrm{B}}$ and $L_{\mathrm{G}}$ and a value of $L_{\mathrm{C}}$ computed from an ephemeris.

\section{Heliocentric gravitation constant, GM $_{\mathrm{S}}$}

To adhere with historical definitions, the value for $G M_{\mathrm{S}}$ should be consistent with $a u$ using the equation $G M_{\mathrm{S}}=a u^{3} k^{2} / D^{2}$. The value for $G M_{\mathrm{S}}$ listed in Table 1 is taken from the Folkner et al. (2008) fit to the DE421 ephemerides. It was not derived using the value of $a u$ listed in Table 1 but the TDB-compatible value of $G M_{\mathrm{S}}$ in Table 1 is consistent with the value of $a u$ in Table 1 (Pitjeva and Standish 2009) to within the errors of the estimate. Both TCB-compatible and TDB-compatible values are provided.

Since it is now possible to estimate directly a value for $G M_{\mathrm{S}}$, it is expected that a recommendation will be discussed at the 2012 IAU General Assembly to change the historical relationship between $G M_{\mathrm{S}}, k$, and $a u$. Should this occur, the CBE list of the NSFA WG would be changed accordingly.

\section{Equatorial radius of the Earth, $a_{\mathrm{E}}$}

The equatorial radius of the Earth, $a_{\mathrm{E}}$, is taken from Burša et al. (1998) and was included in the International Association of Geodesy (IAG) Special Commission 3 (SC3) Report in Groten (2000). Note that $a_{\mathrm{E}}$ is a "zero tide" value. See the IERS Conventions for more explanation of the terminology "zero tide". Note that although the value is listed as TT-compatible, the TCG-compatible value is equivalent to the number of decimal places listed.

\section{Dynamical form factor, $J_{2}$}

The dynamical form factor, $J_{2}$, is taken from the International Association of Geodesy (IAG) Special Commission 3 (SC3) Report provided by Groten (2000). Note that $J_{2}$ is a "zero tide" value. See the IERS Conventions for more explanation of the terminology "zero tide". 
Time rate of change in $J_{2}, \dot{J}_{2}$

The time rate of change in $J_{2}, \dot{J}_{2}$, is a value adopted in IAU 2006 Resolution B1 that is consistent with the adopted IAU 2006 precession model. The value is taken from Capitaine et al. (2005). A discussion about the uncertainty can be found in Bourda and Capitaine (2004) and in Hilton et al. (2006) while a discussion about the components of the $J_{2}$ variation can be found in Cheng and Tapley (2004) with a 2008 AGU update (2008AGUFM.G33A0673C).

\section{Geocentric gravitation constant, GM $\mathrm{E}$}

The geocentric gravitation constant, $G M_{\mathrm{E}}$, is taken from Ries et al. (1992). TCBcompatible, TT-compatible, and TDB-compatible values are provided.

\section{Potential of the geoid, $W_{0}$}

The potential of the geoid, $W_{0}$, is taken from the International Association of Geodesy (IAG) Special Commission 3 (SC3) Report provided by Groten (2000).

\section{Nominal mean angular velocity of the Earth, $\omega$}

The nominal mean angular velocity of the Earth, $\omega$, is taken from the IAG SC3 Report provided by Groten (2000). Note that $\omega$ is a nominal value chosen to have the number of significant digits limited to those for which the value can be considered constant. Note that although the value is listed as TT-compatible, the TCG-compatible value is equivalent to the number of decimal places listed.

Ratio of the mass of the Moon to the Earth, $M_{\mathrm{M}} / M_{\mathrm{E}}$

The value for the ratio of the mass of the Moon to the mass of the Earth, $M_{\mathrm{M}} / M_{\mathrm{E}}$, is taken from Pitjeva and Standish (2009). Note that this is equivalent to $M_{\mathrm{E}} / M_{\mathrm{M}}=81.3005678 \pm$ $2.7 \times 10^{-6}$.

Ratio of the mass of the Sun to Mercury, $M_{\mathrm{S}} / M_{\mathrm{Me}}$

The value for the ratio of the mass of the Sun to the mass of Mercury, $M_{\mathrm{S}} / M_{\mathrm{Me}}$, is taken from Anderson et al. (1987).

Ratio of the mass of the Sun to Venus, $M_{\mathrm{S}} / M_{\mathrm{Ve}}$

The value for the ratio of the mass of the Sun to the mass of Venus, $M_{\mathrm{S}} / M_{\mathrm{Ve}}$, is taken from Konopliv et al. (1999).

Ratio of the mass of the Sun to Mars, $M_{\mathrm{S}} / M_{\mathrm{Ma}}$

The value for the ratio of the mass of the Sun to the mass of Mars, $M_{\mathrm{S}} / M_{\mathrm{Ma}}$, is taken from Konopliv et al. (2006).

Ratio of the mass of the Sun to Jupiter, $M_{\mathrm{S}} / M_{\mathrm{J}}$

The value for the ratio of the mass of the Sun to the mass of Jupiter, $M_{\mathrm{S}} / M_{\mathrm{J}}$, is taken from Jacobson et al. (2000).

Ratio of the mass of the Sun to Saturn, $M_{\mathrm{S}} / M_{\mathrm{Sa}}$

The value for the ratio of the mass of the Sun to the mass of Saturn, $M_{\mathrm{S}} / M_{\mathrm{Sa}}$, is taken from Jacobson et al. (2006). 
Ratio of the mass of the Sun to Uranus, $M_{\mathrm{S}} / M_{\mathrm{U}}$

The value for the ratio of the mass of the Sun to the mass of Uranus, $M_{\mathrm{S}} / M_{\mathrm{U}}$, is taken from Jacobson et al. (1992).

Ratio of the mass of the Sun to Neptune, $M_{\mathrm{S}} / M_{\mathrm{N}}$

The value for the ratio of the mass of the Sun to the mass of Neptune, $M_{\mathrm{S}} / M_{\mathrm{N}}$, is taken from Jacobson (2009).

Ratio of the mass of the Sun to (134340) Pluto, $M_{\mathrm{S}} / M_{\mathrm{P}}$

The value for the ratio of the mass of the Sun to the mass of (134340) Pluto, $M_{\mathrm{S}} / M_{\mathrm{P}}$, is taken from Tholen et al. (2008). Note that this is equivalent to $M_{\mathrm{P}} / M_{\mathrm{S}}=7.32247 \times 10^{-9} \pm$ $0.00150 \times 10^{-9}$.

Ratio of the mass of the Sun to (136199) Eris, $M_{\mathrm{S}} / M_{\text {Eris }}$

The value for the ratio of the mass of the Sun to the mass of (136199) Eris, $M_{\mathrm{S}} / M_{\mathrm{Eris}}$, is taken from Brown and Schaller (2007). Note that this is equivalent to $M_{\text {Eris }} / M_{\mathrm{S}}=8.396$ $\times 10^{-9} \pm 0.100 \times 10^{-9}$.

Ratio of the mass of (1) Ceres to the Sun, $M_{\text {Ceres }} / M_{\mathrm{S}}$

The value for the ratio of the mass of (1) Ceres to the mass of the Sun, $M_{\text {Ceres }} / M_{\mathrm{S}}$, is taken from Pitjeva and Standish (2009).

Ratio of the mass of (2) Pallas to the Sun, $M_{\text {Pallas }} / M_{\mathrm{S}}$

The value for the ratio of the mass of (2) Pallas to the mass of the Sun, $M_{\text {Pallas }} / M_{\mathrm{S}}$, is taken from Pitjeva and Standish (2009).

Ratio of the mass of (4) Vesta to the Sun, $M_{\mathrm{Vesta}} / M_{\mathrm{S}}$

The value for the ratio of the mass of (4) Vesta to the mass of the Sun, $M_{\mathrm{Vesta}} / M_{\mathrm{S}}$, is taken from Pitjeva and Standish (2009).

Obliquity of the ecliptic at J2000.0, $\epsilon_{\mathrm{J} 2000}$

The obliquity of the ecliptic at J2000.0, $\epsilon_{\mathrm{J} 2000}$, is from the Hilton et al. (2006) report from the IAU Working Group on precession and the ecliptic. This value is taken from the P03 precession model of Capitaine et al. (2003) and was adopted in IAU 2006 Resolution B1. The value was determined by Chapront et al. (2002) using lunar laser ranging observations. Note that $\epsilon_{\mathrm{J} 2000}$ is a component of the IAU 2006 precession model that includes expressions that are time dependent.

\section{Future of the working group}

Even with the dedication and effort that the Working Group has displayed over the last triennium, there is still additional work that is yet to be completed in setting up the process of determining and maintaining a list of CBEs. In general, these efforts can be split along the lines of procedural and scientific efforts. 


\subsection{Procedural efforts}

In order to ensure the integrity of the CBEs, a formal procedure for the adoption of new values and the archiving of older versions of the CBEs is under development. This involves the procedure for proposing, discussing and deciding on whether new values should be adopted. Important factors to consider in this process include:

- ensuring that the value is in an article in a refereed journal;

- whether the new value is an improvement over the old;

- whether the new value is provisional;

- the length of time expected before a new estimate will become available;

- whether the article discusses how the current value compares with the old.

In addition, sufficient documentation will need to be kept to ensure that the current status of the CBEs can be determined and the existing situation of past sets of CBEs can also be recreated. Establishing these procedures for changing the CBEs and properly documenting the lists will be a primary goal of the WG during this triennium.

As recommended by the IAU Resolution, the CBEs will be provided to the astronomical community as an electronic document. This is the best way to facilitate the most accurate estimates being available to the user community in a timely fashion. Several different options are being considered. Although it is unlikely to be hosted on the official IAU web pages, it might be reasonable to host the web page from the IAU Division I or one of the IAU Commission web pages.

At the 2012 IAU General Assembly in Beijing, it is expected that the WG will be dissolved. However, it is desirable to have the service of maintaining a list of CBEs for the IAU and the astronomical community continue within the IAU. IAU Division I has suggested that the IAU change its by-laws to permit standing working groups. A standing working group would allow for the work of the NSFA WG to continue without concern for the possibility of needing to dissolve and reconstitute the body.

\subsection{Scientific efforts}

The WG will continue to provide the astronomical community with the current best estimates. This will involve keeping up with improvements due to better measurements, experiments, space missions, etc. while being mindful of the limitations in other constants. It is expected that alternative methods for determining masses of asteroids and values provided by the geodetic community will play important roles.

There are also some specific issues that the WG will be addressing. For instance, the Working Group could not reach a unanimous consensus on the future of the astronomical unit. It is expected that this issue will be resolved by the preparation of a draft resolution to be presented at the 2012 IAU General Assembly.

Acknowledgments The authors would like to thank the anonymous reviewer for the careful reading and the helpful suggestions.

Open Access This article is distributed under the terms of the Creative Commons Attribution Noncommercial License which permits any noncommercial use, distribution, and reproduction in any medium, provided the original author(s) and source are credited. 


\section{Appendix}

The following is the text of IAU 2009 Resolution B2.

1. The XXVII International Astronomical Union General Assembly,

\section{Considering}

1. the need for a self-consistent set of accurate numerical standards for use in astronomy,

2. that improved values of astronomical constants have been derived from recent observations and published in refereed journals, and

3. that conventional values have been adopted by IAU GA 2000 and IAU GA 2006 resolutions for a number of astronomical quantities,

\section{Recognizing}

1. the continuing need for a set of Current Best Estimates (CBEs) of astronomical numerical constants, and

2. the need for an operational service to the astronomical community to maintain the CBEs

\section{Recommends}

1. that the list of previously published constants compiled in the report of the Working Group on Numerical Standards for Fundamental Astronomy (see http://maia.usno.navy. mil/NSFA/CBE.html) be adopted as the IAU (2009) System of Astronomical Constants.

2. that Current Best Estimates of Astronomical Constants be permanently maintained as an electronic document,

3. that, in order to ensure the integrity of the CBEs, IAU Division I develop a formal procedure to adopt new values and archive older versions of the CBEs, and

4. that the IAU establish within IAU Division I a permanent body to maintain the CBEs for fundamental astronomy.

\section{References}

Anderson, J.D., Colombo, G., Esposito, P.B., Lau, E.L., Trager, G.B.: The mass gravity field and ephemeris of Mercury. Icarus 71, 337-349 (1987)

Bourda, G., Capitaine, N.: Precession, nutation and space geodetic determination of the Earth's variable gravity field. Astron. Astrophys. 428, 691-702 (2004)

Brown, M.E., Schaller, E.L.: The Mass of Dwarf Planet Eris. Science 316, 1585 (2007). doi:10.1126/science. 1139415

Burša, M., Kouba, J., Raděj, K., True, S.A., Vatrt, V., Vojtišková, M.: Mean earth's equipotential surface from Topex/Poseidon altimetry. Studia Geoph. Et Geod. 42, 459-466 (1998). doi:10.1023/A:1023356803773

Capitaine, N., Guinot, B., McCarthy, D.D.: Definition of the Celestial Ephemeris Origin and of UT1 in the International Celestial Reference Frame. Astron. Astrophys. 355, 398-405 (2000)

Capitaine, N., Wallace, P., Chapront, J.: Expressions for IAU 2000 precession quantities. Astron. Astrophys. 412, 567-586 (2003)

Capitaine, N., Wallace, P.T., Chapront, J.: Improvement of the IAU 2000 precession model. Astron. Astrophys. 432, 355-367 (2005)

Chapront, J., Chapront-Touzé, M., Francou, G.: A new determination of lunar orbital parameters, precession constant and tidal acceleration from LLR measurements. Astron. Astrophys. 387, 700-709 (2002). doi:10.1051/0004-6361:20020420

Cheng, M., Tapley, B.D.: Variations in the Earth's oblateness during the past 28 years. J. Geophys. Res. 109, B09402 (2004). doi:10.1029/2004JB003028

CODATA, http://physics.nist.gov/cuu/Constants/index.html (2006)

Fairhead, L., Bretagnon, P.: An Analytical Formula for the Time Transformation TB-TT. Astron. Astrophys. 229, 240-247 (1990) 
Folkner, W.M., Williams, J.G., Boggs, D.H.: The planetary and Lunar Ephemeris DE 421. Memorandum IOM 343R-08-003, (2008)

Fukushima, T.: Report on Astronomical Constants. In: Johnston, K.J., McCarthy, D.D., Luzum, B.J., Kaplan, G.H. (eds.) Proceedings of IAU Colloquium 180, pp. 417-427. (2000)

Fukushima, T.: Report on Astronomical Constants. In: Rickman, H. Highlights of Astronomy, Vol. 12, pp. 107-112. International Astronomical Union, 2000, Paris (2002)

Gauss, C.F.: Theory of the Motion of the Heavenly Bodies Moving About the Sun in Conic Sections. p. 2 Little, Brown, and Company, Boston (1857)

Groten, E.: Geodesists Handbook 2000, part 4, http://www.gfy.ku.dk/ iag/HB2000/part4/groten.htm. See also Parameters of Common Relevance of Astronomy, Geodesy, and Geodynamics. J. Geod. 74, 134-140 (2000)

Hilton, J.L., Capitaine, N., Chapront, J., Ferrandiz, J.M., Fienga, A., Fukushima, T., Getino, J., Mathews, P., Simon, J.-L., Soffel, M., Vondrak, J., Wallace, P., Williams, J.: Report of the International Astronomical Union Division I Working Group on Precession and the Ecliptic. Celest. Mech. Dyn. Astron. 94, 351-367 (2006). doi:10.1007/s10569-006-0001-2

IERS Conventions, McCarthy, D.D., Petit, G.: IERS Technical Note 32, Verlag des Bundesamts für Kartographie und Geodäsie, Frankfurt am Main, 127 pp. (2003)

International Astronomical Union (IAU). In: Proceedings of the Sixteenth General Assembly. Transactions of the IAU, XVIB, p. 31, pp. 52-66 (1976)

International Astronomical Union (IAU): Proceedings of the Twenty-Fourth General Assembly. Transactions of the IAU, XXIVB, pp. 34-57 (2000)

International Astronomical Union (IAU), Proceedings of the Twenty-Sixth General Assembly. Transactions of the IAU, XXVIB (2006)

Irwin, A., Fukushima, T.: A numerical time ephemeris of the Earth. Astron. Astrophys. 348, 642-652 (1999)

Jacobson, R.A.: The Orbits of the Neptunian Satellites and the Orientation of the Pole of Neptune. Astron. J. 137, 4322-4329 (2009). doi:10.1088/0004-6256/137/4322

Jacobson, R.A., Campbell, J.K., Taylor, A.H.: The masses of Uranus and its Major Satellites from Voyager Tracking Data and Earth-based Uranian Satellite Data. Astron. J. 103(6), 2068-2078 (1992)

Jacobson, R.A., Haw, R.J., McElrath, T.P., Antreasian, P.G.: A Comprehensive Orbit Reconstruction for the Galileo Prime Mission in the J2000 System. J. Astronaut. Sci. 48(4), 495-516 (2000)

Jacobson, R.A., Antreasian, P.G., Bordi, J.J., Criddle, K.E., Ionasescu, R., Jones, J.B., Mackenzie, R.A., Pelletier, F.J., Owen, W.M. Jr., Roth, D.C., Stauch, J.R.: The Gravity Field of the Saturnian System from Satellite Observations and Spacecraft Tracking Data. Astron. J. 132(6), 2520-2526 (2006)

Klioner, S.A., Capitaine, N., Folkner, W., Guinot, B., Huang, T.-Y., Kopeikin, S., Pitjeva, E., Seidelmann, P. K., Soffel, M.: Units of relativistic time scales and associated quantities. In: Klioner, S., Seidelmann, P.K., Soffel M. (eds.) Relativity in Fundamental Astronomy: Dynamics, Reference Frames, and Data Analysis, Proceedings of the International Astronomical Union Symposium No. 261, 2009, Cambridge University Press, pp. 79-84. (2010) doi:10.1017/S1743921309990184

Konopliv, A.S., Banerdt, W.B., Sjogren, W.L.: Venus Gravity: 180th Degree and Order Model. Icarus 139, 3-18 (1999)

Konopliv, A.S., Yoder, C.F., Standish, E.M., Yuan, D.N., Sjogren, W.L.: A global solution for the Mars static and seasonal gravity, Mars Orientation, Phobos and Deimos masses, and Mars ephemeris. Icarus 182(1), 23-50 (2006)

Mohr, P.J., Taylor, B.N.: CODATA recommended values of the fundamental physical constants: 1998. Rev. Mod. Phys. 72, 351-495 (2000)

Mohr, P.J., Taylor, B.N., Newell, D.B.: The CODATA recommended values of the fundamental physical constants: 2006. Rev. Mod. Phys. 80, 633-730 (2008)

Petit, G.: Report of the BIPM/IAU joint committee on relativity for space-time reference systems and metrology. In: Johnston, K.J., McCarthy, D.D., Luzum, B.J., Kaplan, G.H. (eds.) Proceedings of IAU Colloquium 180, U.S. Naval Observatory, Washington, D.C. pp. 275-282, (2000)

Pitjeva, E.V., Standish, E.M.: Proposals for the masses of the three largest asteroids, the Moon-Earth mass ratio and the Astronomical Unit. Celest. Mech. Dyn. Astron. 103, 365-372 (2009). doi:10.1007/ s10569-009-9203-8

Ries, J.C., Eanes, R.J., Shum, C.K., Watkins, M.M.: Progress in the determination of the gravitational coefficient of the Earth. Geophys. Res. Lett. 19(6), 529-531 (1992)

Standish, E.M: Report of the IAU WGAS Sub-group on Numerical Standards. In: Appenzeller, I. Highlights in Astronomy, Vol. 10, pp. 180-184. International Astronomical Union, 1994, (1995)

Tholen, D.J., Buie, M.W., Grundy, W.: Masses of Nix and Hydra. Astron. J. 135(3), 777-784 (2008) 